\title{
Enhanced Neutrophil Extravasation and Rapid Progression of Proteoglycan-Induced Arthritis in TSG-6-Knockout Mice
}

\author{
Sándor Szántó, Tamás Bárdos, István Gál, Tibor T. Glant, and Katalin Mikecz
}

\begin{abstract}
Objective. To gain insight into the mechanisms of the antiinflammatory effect of tumor necrosis factor $\alpha$ (TNF $\alpha$ )-induced protein 6 (Tnfip6) in arthritis, using Tnfip6-deficient animals.

Methods. TNF $\alpha$-stimulated gene 6 (TSG-6) coding for Tnfip6 was disrupted. Tnfip6-deficient mice were backcrossed into proteoglycan-induced arthritis (PGIA)-susceptible BALB/c mice, and arthritis was induced by systemic immunization with cartilage proteoglycan (PG). Thioglycollate-induced sterile peritonitis was also assessed, to monitor the early events of neutrophil extravasation in wild-type and Tnfip6deficient mice in the presence or absence of treatment with recombinant murine Tnfip6.
\end{abstract}

Results. The onset of PGIA was similar, but progression and severity were significantly greater, in Tnfip6-deficient mice compared with wild-type BALB/c mice. However, this was not associated with enhanced $T$ or B cell responses to cartilage PGs, but rather, an early and more extensive infiltration of the synovium with neutrophil leukocytes was the most prominent histopathologic feature of PGIA in Tnfip6-deficient mice. This was accompanied by elevated serum levels of interleukin- 6 and amyloid $A$, and significantly increased activities of the enzymes plasmin, myeloperoxidase, and neutrophil elastase in the inflamed paw joints of Tnfip6null mice, when compared with that of the wild-type

Supported by the Arthritis Foundation, Greater Chicago Chapter and, in part, by grants from the National Institutes of Health (AR-40310, AR-45652, and AR-051163).

Sándor Szántó, MD (current address: University of Debrecen, Debrecen, Hungary), Tamás Bárdos, MD (current address: University of Pécs, Pécs, Hungary), István Gál, MD (current address: University of Debrecen, Debrecen, Hungary), Tibor T. Glant, MD, PhD, Katalin Mikecz, MD, PhD: Rush University Medical Center, Chicago, Illinois. Address correspondence and reprint requests to Katalin Mikecz, MD, PhD, Section of Biochemistry and Molecular Biology, Rush University Medical Center, Cohn Research Building, Room 712, 1735 West Harrison Street, Chicago, IL 60612. E-mail: Katalin_Mikecz@rsh.net. form May 5, 2004 littermates. Loss of control over several components of inflammation resulted in extensive and rapid cartilage degradation, bone erosion, joint ankylosis, and deformities in Tnfip6-null animals. In support of the antiinflammatory effect of Tnfip6 via the inhibition of polymorphonuclear (PMN) cell efflux, neutrophil invasion during thioglycollate-induced peritonitis was 2-fold higher in Tnfip6-deficient animals than in wild-type animals, but was dramatically suppressed by intravenous injection of recombinant murine Tnfip6.

Conclusion. Tnfip6 is a multifunctional antiinflammatory protein that is produced at the site of inflammation and can be retained by the hyaluronanrich extracellular matrix. A major effect of Tnfip6 is the inhibition of the extravasation of PMN cells, predominantly neutrophils, into the site of inflammation, most likely via a CD44/hyaluronan/Tnfip6-mediated blocking mechanism.

Tumor necrosis factor $\alpha(\mathrm{TNF} \alpha)$-induced protein 6 (Tnfip6), the secreted product of TNF $\alpha$ stimulated gene 6 (TSG-6), is a member of the hyaladherin superfamily of hyaluronan (HA) binding proteins $(1,2)$. The constitutive expression of Tnfip6 is very low, but virtually all cell types of mesenchyme origin can produce Tnfip6 in response to proinflammatory stimuli (2-5). Tnfip6 can be detected in large quantities in synovial fluid samples from inflamed joints, but not in normal healthy joints (3). Expression of Tnfip6 is upregulated in the inflamed synovial tissue of patients with rheumatoid arthritis or osteoarthritis (5), indicating that the in vivo production of this protein requires a milieu enriched in proinflammatory mediators.

Recombinant murine Tnfip6 (rMuTnfip6) has been shown to have a therapeutic effect on both collagen-induced arthritis (6) and proteoglycan-induced arthritis (PGIA) (7), and exhibits antiinflammatory and chondroprotective properties in antigen-induced arthritis (7). A chondroprotective effect could also be 
achieved in antigen-induced arthritis by cartilagespecific constitutive expression of the Tnfip6 transgene $(8)$.

Tnfip6 forms a stable complex with inter- $\alpha$ trypsin inhibitor $(\mathrm{I} \alpha \mathrm{I})(9,10)$, a major serine protease inhibitor in serum, and potentiates the inhibitory activity of $\mathrm{I} \alpha \mathrm{I}$ against plasmin. The inhibitory effect of the Tnfip6-I $\alpha$ I complex appears to be specific for plasmin, since no increase was observed in the inhibitory activity against other serine proteases (10). Since plasmin is a key activator of matrix-degrading metalloproteases, it has been postulated that Tnfip6 exerts its antiinflammatory and chondroprotective effects through the inactivation of the metalloprotease network $(7,9,10)$.

To further elucidate the antiinflammatory properties of Tnfip6, an air-pouch model of acute inflammation was used. Injection of recombinant human Tnfip6 (rHuTnfip6) together with a stimulant (carrageenan or zymosan) into the air pouch of mice resulted in significant reductions in the number of emigrated neutrophils, as compared with air pouches injected with the stimulant only $(10,11)$. With the use of mutant forms of rHuTnfip6, which has a reduced ability to bind HA via its Link module, it has been shown that most of the antiinflammatory activity of Tnfip6 resides within its Link module domain (11).

The primary objective of the present study was to determine whether the lack of Tnfip6 increases the predisposition of mice to develop a systemic autoimmune form of experimentally induced arthritis such as PGIA. For this purpose, we backcrossed Tnfip6deficient mice (also referred to as Tnfip6-null, Tnfip6 ${ }^{-1-}$, and Tnfip6-knockout mice) (originally created in the $129 \mathrm{~Sv} / \mathrm{C} 57 \mathrm{BL} / 6$ mixed background) into $\mathrm{BALB} / \mathrm{c}$, a strain that exhibits genetic susceptibility to PGIA (12-14). Tnfip6-null BALB/c mice developed arthritis with greater severity than that observed in the wild-type littermates. Moreover, the development of PGIA in Tnfip6-deficient mice was associated with an overwhelming influx of neutrophils into the joints, which was rapidly followed by destruction of articular cartilage, erosion of bone, and ankylosis. Accelerated extravasation of neutrophil granulocytes in the absence of Tnfip6 was not specific to the manifestations of arthritis, since significantly greater influx of neutrophils was also observed in thioglycollate-induced sterile peritonitis, and we found that this could be normalized to the levels in the wild-type littermates by exogenous addition of rMuTnfip6.

\section{MATERIALS AND METHODS}

Generation of Tnfip6-null mice. Tnfip6 is a $20.3-\mathrm{kb}-$ long gene on mouse chromosome 2 (4). Tnfip6-deficient mice were generated by disrupting exon 1 with the $N e o^{\mathrm{r}}$-poly(A) cassette, creating a translation stop codon at 94 bp downstream of the translation start site in the Tnfip6 gene (15). As described earlier, the only phenotype abnormality in Tnfip6deficient mice is the infertility of the Tnfip $6^{-1-}$ females (15). Using a marker-assisted speed-backcrossing protocol, heterozygous (Tnfip $6^{+-}$) males were mated with wild-type $\mathrm{BALB} / \mathrm{c}$ females. Males were genotyped for the presence of the Neo gene; the heterozygous males were further tested for genomic composition using a total of 147 simple sequencelength polymorphic markers $(16,17)$. Tnfip $6^{+/-}$males with the most extensive BALB/c background were selected for subsequent backcrosses. This process was repeated 6 times until homogeneity of the BALB/c genome was achieved, and only a small region on chromosome 2 (harboring the Tnfip6 gene) was a non-BALB/c locus.

The Tnfip6 mutant colony has been maintained by intercrossing Tnfip $6^{+/-}$females with Tnfip $6^{+/-}$males, and has been expanded to generate sufficient numbers of Tnfip6deficient (Tnfip $\left.6^{-/-}\right)$and wild-type $\left(\operatorname{Tnfip} 6^{+/+}\right)$littermates for all experiments. The animal procedures were approved by the Animal Care and Use Committee of Rush University Medical Center. All mice were housed under standard conditions.

Enzyme-linked immunosorbent assays (ELISAs) for rMuTnfip6 and Tnfip6. Recombinant murine Tnfip6 was purified on an HA-coupled EAH-Sepharose column from supernatants of Chinese hamster ovary cells (CHO-K1; American Type Culture Collection, Manassas, VA) that were stable transfected with the Lonza pEE14.1 vector (Lonza Biologics, Slough, Berkshire, UK) containing a full-length $(1,654 \mathrm{bp})$ mouse Tnfip6 complementary DNA clone as described previously (7). Further purification was performed using reversephase high-pressure liquid chromatography as described for rHuTSG-6/rHuTNFIP6 (18). The purified product was then lyophilized and dissolved in phosphate buffered saline (PBS) $(\mathrm{pH} 7.4)$ at $1 \mathrm{mg} / \mathrm{ml}$ concentration. For in vivo treatment of mice, the rMuTnfip6 solution was sterilized by passing it through a $0.2-\mu \mathrm{m}$-pore-size sterile-syringe filter.

The rat monoclonal antibody (mAb), A38, recognizing the Link module domains of both human and mouse Tnfip6, has been described previously (19). A sandwich ELISA was developed using purified A38 mAb for capture, and biotinylated TSG-6-CR21 polyclonal rabbit antibody $(7,8)$ was used for detection of Tnfip6 in serially diluted serum samples. Peroxidase-conjugated streptavidin (Zymed, San Francisco, CA) followed by $o$-phenylenediamine and hydrogen peroxide were used for the colorimetric detection of plate-bound Tnfip6, and rMuTnfip6 served as a reference standard.

Immunization with cartilage proteoglycan (PG) and assessment of arthritis. PG was isolated from human osteoarthritic cartilage using a standard protocol (20), and the glycosaminoglycan side chains were depleted by subsequent digestions of PG with endo- $\beta$-galactosidase and chondroitinase ABC (Seikagaku America, Falmouth, MA) as described previously (20,21). Tnfip6 $6^{+/+}$and Tnfip6 ${ }^{-1-}$ male and female mice, at 10-12 weeks of age, were immunized intraperitoneally with $100 \mu \mathrm{g}$ PG protein that was dissolved in $100 \mu \mathrm{l}$ sterile PBS 
and emulsified with $1 \mathrm{mg}$ of the adjuvant dimethyldioctadecylammonium bromide (Sigma-Aldrich, St. Louis, MO) as previously described $(14,21)$. The same doses of antigen and adjuvant were injected on days 21 and 42, and, if necessary, on day 63.

The paws of all immunized mice were examined twice a week until day 21 , and thereafter examined daily to record abnormalities due to arthritic changes of the joints. The first appearance of joint swelling was recorded as the day of the onset of arthritis. A standard scoring system, based on the presence of swelling and redness and having a range of $0-4$ for each paw (thus resulting in a possible maximum score of 16 for each animal), was used for the assessment of disease severity $(12,14,22,23)$. Ankylosis in the peripheral joints (knee and ankle) was defined as reduced joint mobility due to bone deformities and periarticular stiffness.

Nonimmunized and PG-immunized arthritic wild-type and Tnfip $6^{-1-}$ mice were killed, and the limbs were photographed and subjected to radiographic analysis using a Hewlett Packard Faxitron X-ray System (85 KV for 18 seconds, model 43855A; Hewlett Packard, McMinnville, OR) and highresolution Kodak X-Omat film (Eastman Kodak, Rochester, NY). The limbs were then dissected, fixed, decalcified, embedded in paraffin, and sectioned. Tissue sections were stained with hematoxylin and eosin for histopathologic examination.

Measurements of antigen-specific $T$ cell responses, antibodies, and cytokines. During the immunization period (once a week) and at the end of the experiments, blood samples (for serum) were collected from the retroorbital venous plexus of immunized mice. Spleen cells were harvested at the end of the experiments. PG-specific antibodies in serum were measured using ELISA, as described previously $(16,17,23)$. Briefly, 96-well Maxisorp plates (Nunc International, Hanover Park, IL) were coated with human or mouse cartilage PG $(0.1 \mu \mathrm{g}$ protein/well), the free binding sites were blocked with $1 \%$ fat-free milk in PBS, and PG-specific antibodies were measured in serially diluted serum samples of PG-immunized mice. The total serum concentrations of antiPG antibodies (IgG, IgM, and IgA) were determined using peroxidase-conjugated goat anti-mouse $\mathrm{IgG}, \mathrm{IgM}$, and $\mathrm{IgA}$ antibodies (Accurate Chemical \& Scientific, Westbury, NY), and PG-specific IgG isotypes were detected with rat $\mathrm{mAb}$ to mouse IgG1 or IgG2a (Zymed) as secondary reagents $(16,23)$. Serum antibody levels were calculated relative to the concentrations of the corresponding mouse IgG isotype standards (all from Zymed) or total mouse serum Ig fractions (SigmaAldrich) applied to the ELISA plates $(17,23)$.

Antigen-specific $\mathrm{T}$ cell responses were determined in quadruplicate samples of spleen cells $\left(3 \times 10^{5}\right.$ cells/well $)$ cultured in 96-well plates in the presence of $25 \mu \mathrm{g}$ PG protein $/ \mathrm{ml}$ culture medium. Antigen-specific interleukin-2 (IL-2) production was measured in the culture supernatants 48 hours later, using the CTLL-2 bioassay, and T cell proliferation was assessed on day 5 by incorporation of ${ }^{3} \mathrm{H}$-thymidine $(16,17,20,23)$. The antigen-specific $\mathrm{T}$ cell response was expressed as the stimulation index, a ratio of incorporated ${ }^{3} \mathrm{H}$-thymidine (in counts per minute) in antigen-stimulated spleen cell cultures relative to the cpm measured in nonstimulated cultures. Antigen-specific production of interferon- $\gamma$ and IL-4 was measured in cell culture supernatants $\left(3 \times 10^{6}\right.$ cells/ml) on day 4 using capture ELISA methods (BD Pharm- ingen, San Diego, CA, or R\&D Systems, Minneapolis, MN) as previously described $(16,17,21)$. IL- $1 \beta$, IL-6, and TNF $\alpha$ were also measured in the serum samples of PG-immunized animals. Paired $\mathrm{mAb}$ and cytokine standards for ELISA were obtained from BD Pharmingen or R\&D Systems, and the mouse serum amyloid A (SAA) ELISA kit was purchased from Biosource International (Camarillo, CA).

Determination of the activities of enzymes (plasmin, myeloperoxidase, and neutrophil elastase) in joint tissue extracts. Nonarthritic or arthritic hind paws of the animals were snap-frozen in liquid nitrogen and stored at $-80^{\circ} \mathrm{C}$. The frozen paws were homogenized in PBS containing $0.5 \%$ hexadecyl-trimethylammonium bromide, $0.1 \%$ sodium dodecyl sulfate, and 1\% Nonidet P40 (all purchased from SigmaAldrich), sonicated on ice, and cleared by centrifugation. An assay for plasmin activity was performed using the chromogenic substrate tosyl-Gly-Pro-Lys-4-nitranilide acetate (Chromozym PL; Roche, Mannheim, Germany) $(10,11)$.

The accumulation of neutrophils in inflamed paws was monitored by assaying myeloperoxidase activity in the supernatants of paw extracts, with hydrogen peroxide as a substrate and 3,3',5,5'-tetramethylbenzidine as a chromogenic reagent (24). Neutrophil elastase activity was measured using the substrate N-methoxysuccinyl-Ala-Ala-Pro-Val-pNA (Elastin Products, Owensville, MO) (25), in accordance with the manufacturer's instruction. The results of all enzyme assays were normalized to the total protein concentration of the paw extracts, determined by the bicinchoninic acid method (Pierce, Rockford, IL). Myeloperoxidase activity was expressed as the equivalent of the neutrophil numbers found in the peripheral blood of normal BALB/c mice. Colorimetric reactions of serially diluted, purified neutrophil elastase (Elastin Products) and plasmin (Sigma-Adrich) with the specific substrates served as standards for calculation of the activities of the respective enzymes in the tissue extracts.

Neutrophil extravasation in thioglycollate-induced peritonitis in wild-type and Tnfip6-deficient mice, with or without treatment with rMuTnfip6. Peritonitis was induced by intraperitoneal injection of $0.5 \mathrm{ml}$ of $4 \%$ sterile thioglycollate (Becton-Dickinson, Sparks, MD) as described previously $(26,27)$. Peritoneal lavage was performed $0,2,4,6,8,12,24,48$, 72 , and 96 hours after thioglycollate administration. Ten milliliters of ice-cold PBS was injected into the peritoneal cavity of each mouse, and the abdomen was massaged for 2 minutes, after which $4 \mathrm{ml}$ of the lavage fluid was collected.

Leukocytes in the fluid samples were stained with $0.2 \%$ crystal violet (Sigma-Aldrich) in $10 \%$ acetic acid, and then counted in a hemocytometer; total cell numbers were calculated for the 10-ml volume of PBS injected intraperitoneally. Leukocyte differential counts were determined in Giemsastained smears of the cell pellets. The cellular composition of the peritoneal lavage fluid was confirmed by flow cytometry using fluorochrome-labeled mAb to CD45/B220 (B cells), CD3 ( $\mathrm{T}$ cells), and Gr-1 (granulocytes) (all from BD Pharmingen) and $\mathrm{mAb}$ F4/80 to macrophages/monocytes (purchased from Serotec, Raleigh, NC) (21). Separate groups of wild-type and Tnfip6-deficient mice were injected intravenously with $30 \mu \mathrm{g}$ rMuTnfip6 in $60 \mu \mathrm{l}$ PBS 20 minutes before intraperitoneal thioglycollate administration, and the effect of rMuTnfip6 treatment on the cell counts, as well as the types of recruited leukocytes in the peritoneal lavage fluid, were determined at 0 , 
$2,4,8,12,24$, and 48 hours after thioglycollate injection, as described above.

Statistical analysis. Statistical analysis was performed using SPSS software, version 7.5 (SPSS, Chicago, IL). The Mann-Whitney and Wilcoxon tests, or the Student's $t$-test, were used for comparisons between wild-type and Tnfip6deficient mice. The level of significance was set at a $P$ value less than 0.05 .

\section{RESULTS}

Increased incidence and severity of PGIA in Tnfip6-deficient BALB/c mice. Tnfip6-deficient mice and wild-type littermates were monitored for the development of arthritis for 11-15 weeks after the primary immunization with PG. Clinical signs of arthritis first appeared between experimental days 47 and 49 (5-7 days after the third PG injection) in both groups (Figure $1)$. The incidence of PGIA increased more rapidly in the Tnfip6-null mice than in the wild-type mice, and by day 71 (8 days after the fourth PG injection), all of the mutant mice were arthritic, whereas the incidence of arthritis was only $62 \%$ in the wild-type group at this time point (Figure 1A). The disease progressed more rapidly and the arthritic limbs exhibited higher inflammation scores in the Tnfip $6^{-/-}$mice than in the wild-type littermates throughout the entire observation period (Figure 1B). However, there were no differences in disease onset, severity, or incidence between the males and females in either group.

Gross morphology, results of histopathology, and radiographic appearance of the peripheral joints. Histopathologic examination of the hind paw joint sections prepared at the early phase of joint inflammation ( 2 days after onset) revealed more extensive infiltration of the synovial lining by polymorphonuclear (PMN) cells (mostly neutrophils) and a much higher number of neutrophils in the joint exudates of Tnfip6-null mice compared with wild-type mice (Figures $2 \mathrm{~A}$ and B). On day 10 after the onset of PGIA, the histologic assessment showed evidence of inflammatory damage in only the upper zone of articular cartilage in the joints of wildtype mice (Figure 2C), whereas the cartilage had been completely destroyed in the joints of Tnfip6-deficient mice at this time point (Figure 2D).

As expected from the results of our earlier studies that demonstrated a chondroprotective effect of intraarticularly injected rMuTnfip6 (7), the articular cartilage was not protected from the inflammatory attack in mice lacking endogenous Tnfip6. There was no evidence of cartilage loss in the absence of inflammation in Tnfip6-knockout mice (e.g., in the nonarthritic joints
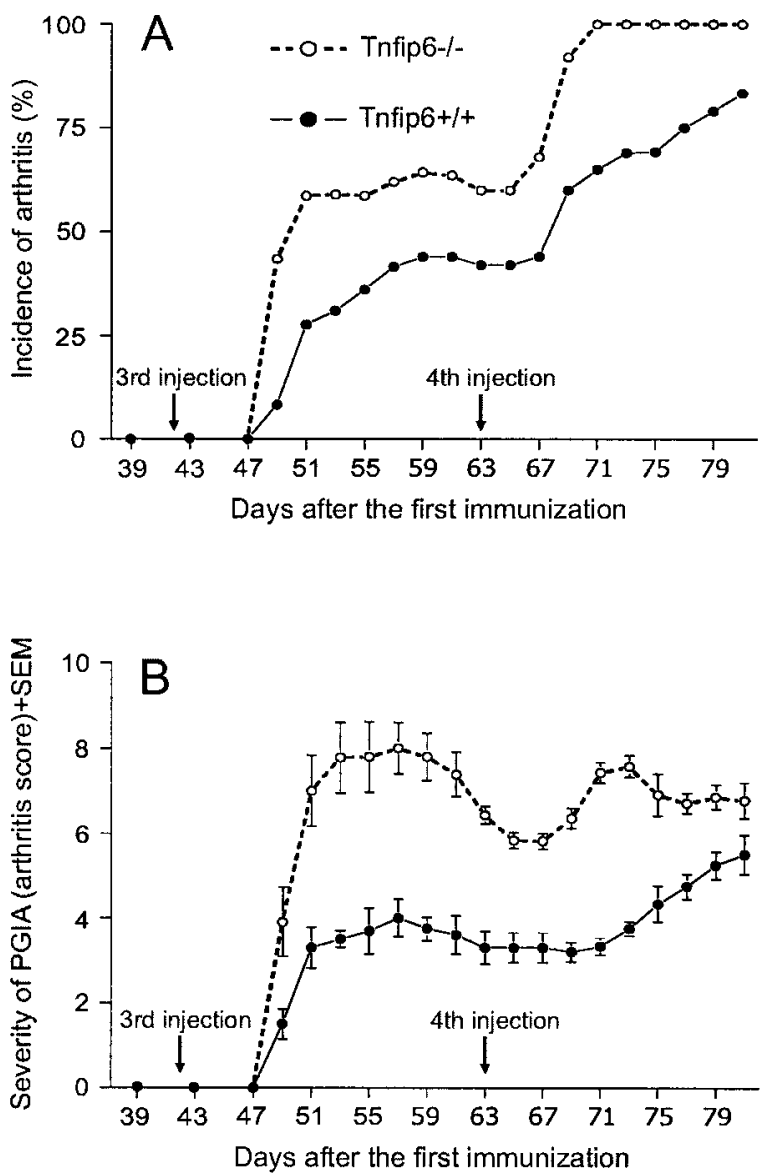

Figure 1. Incidence and severity of proteoglycan-induced arthritis (PGIA) in tumor necrosis factor $\alpha$-induced protein 6 (Tnfip6)deficient $\left(\right.$ Tnfip $\left.^{-/-}\right)$and wild-type $\left(\right.$Tnfip $\left.^{+/+}\right)$BALB/c mice. Arthritis was first detected on day 49 in both groups, i.e., 7 days after the third intraperitoneal immunization with cartilage proteoglycan, but both the incidence (A) and severity (B) of arthritis increased faster and remained higher during the experimental period in the Tnfip6deficient BALB/c mice than in their wild-type littermates. Bars show the mean and SEM. Thirty-five Tnfip $6^{-/-}$and 32 wild-type (Tnfip $6^{+/+}$) mice were used in 3 independent immunization experiments.

of PG-immunized animals [results not shown]), indicating that cartilage destruction did not occur spontaneously, but was triggered by the inflammatory process. Only 10 days after the first appearance of joint swelling, Tnfip6-deficient animals showed histologic signs of reactive fibrosis at the joint margins (Figure 2D), a presumed repair response that usually occurs in wildtype BALB/c mice at a late stage of chronic PGIA, i.e., several weeks after the onset of arthritis (14).

As a result of the rapid progression and increased 


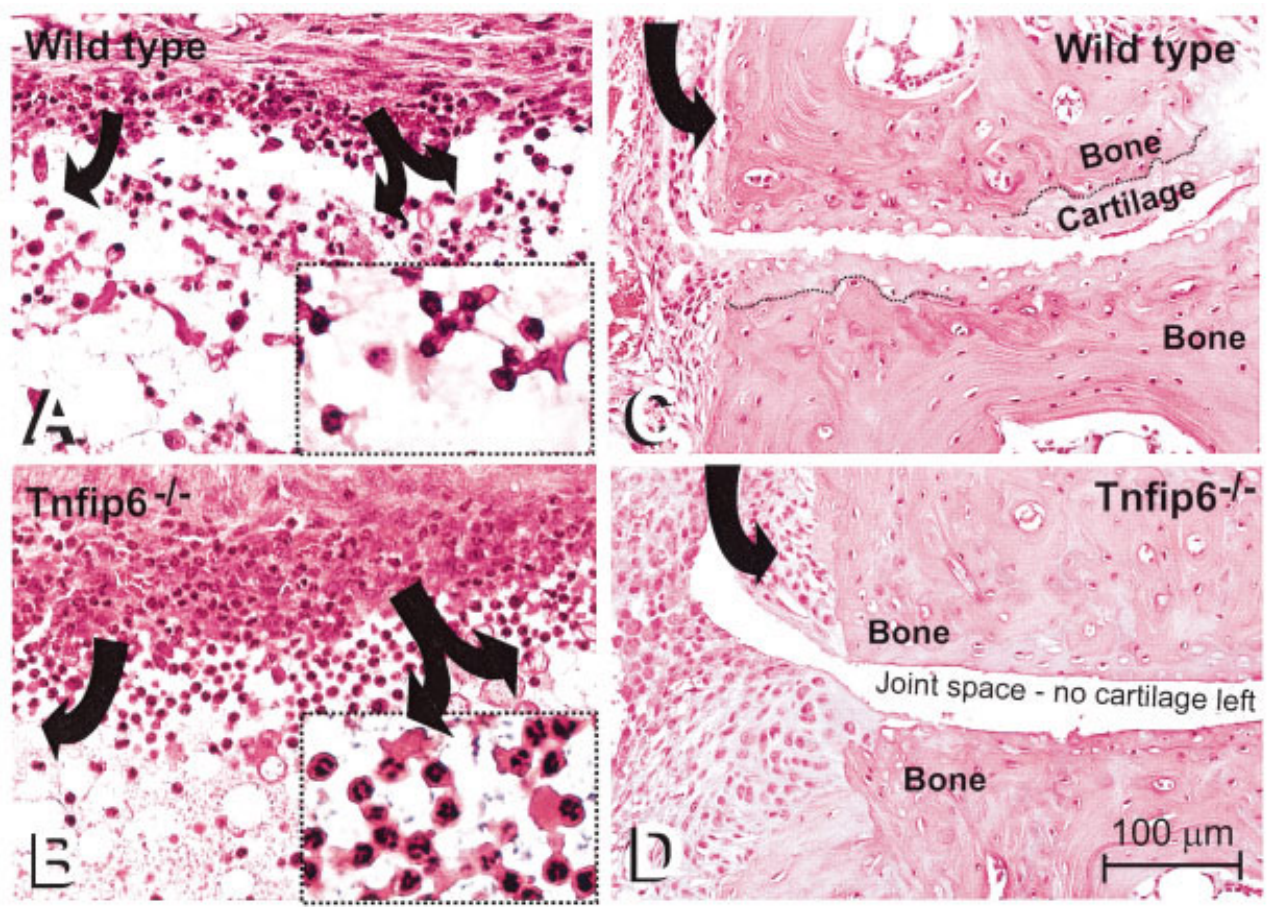

Figure 2. Histopathology of PGIA in wild-type and Tnfip6-deficient BALB/c mice 2 days (A and $\mathbf{B}$ ) and 10 days (C and D) after the onset of arthritis affecting the metatarsophalangeal (MTP) joints of the hind paws. Massive neutrophil invasion dominates the histopathologic picture at the very early stage, and this is notably more pronounced in the synovial lining and joint cavity of the Tnfip6-deficient animal (B) (arrows) than in that of a wild-type littermate (A) (arrows). Insets show high-magnification views of neutrophils at the interface of the synovium and joint cavity. Ten days after the onset of PGIA, cartilage and bone damage are evident in both wild-type and Tnfip $6^{-1-}$ joints $(\mathbf{C}$ and $\mathbf{D})$; however, the articular cartilage has already been completely destroyed in the MTP joint of the Tnfip6-deficient mouse (D). The joint of the Tnfip $6^{-1-}$ mouse also shows histologic evidence of bone erosion and subsequent replacement of bone by a fibrotic tissue (D) (arrow) (compare with arrow in C). (Hematoxylin and eosin stained.) See Figure 1 for other definitions.

severity of PGIA in Tnfip6-deficient animals, massive ankylosis developed 5-7 weeks earlier than in the wildtype littermates. The degree of ankylosis and the extent of joint deformities in Tnfip6-knockout mice far exceeded those ever observed in wild-type BALB/c mice (see Figures 3D and G). The extremely aggressive character of PGIA in Tnfip6-deficient animals was reflected in the high degree of pathologic changes that occurred in the joint shape and in the structure and density of the subchondral bone (e.g., in the metatarsophalangeal joints), as revealed by macroscopic inspection, histology, and radiographic analysis, respectively (Figure 3).

Immune responses and cytokine profiles in PGimmunized Tnfip6-deficient and wild-type BALB/c mice. PGIA is a systemic disease initiated and governed by autoreactive $\mathrm{T}$ cell responses and autoantibodies against the mouse (self) cartilage PG $(13,14,20)$. Therefore, it was important to determine whether the dramatic differences in arthritis severity and in the rate of disease progression between wild-type and Tnfip6-deficient mice (as shown in Figures 1-3) were associated with a more aggressive autoimmune response and/or the production of related cytokines in BALB/c mice lacking Tnfip6. T and B cell responses and cytokine levels were examined at 3 different time points during disease induction and development: in the period prior to onset of arthritis (a few days after the third antigen injection) when the animals were still asymptomatic (Figure 1), at the acute phase (2-3 days after the onset of arthritis), and during the chronic phase ( $>4$ weeks after the onset of arthritis). We did not find significant differences 

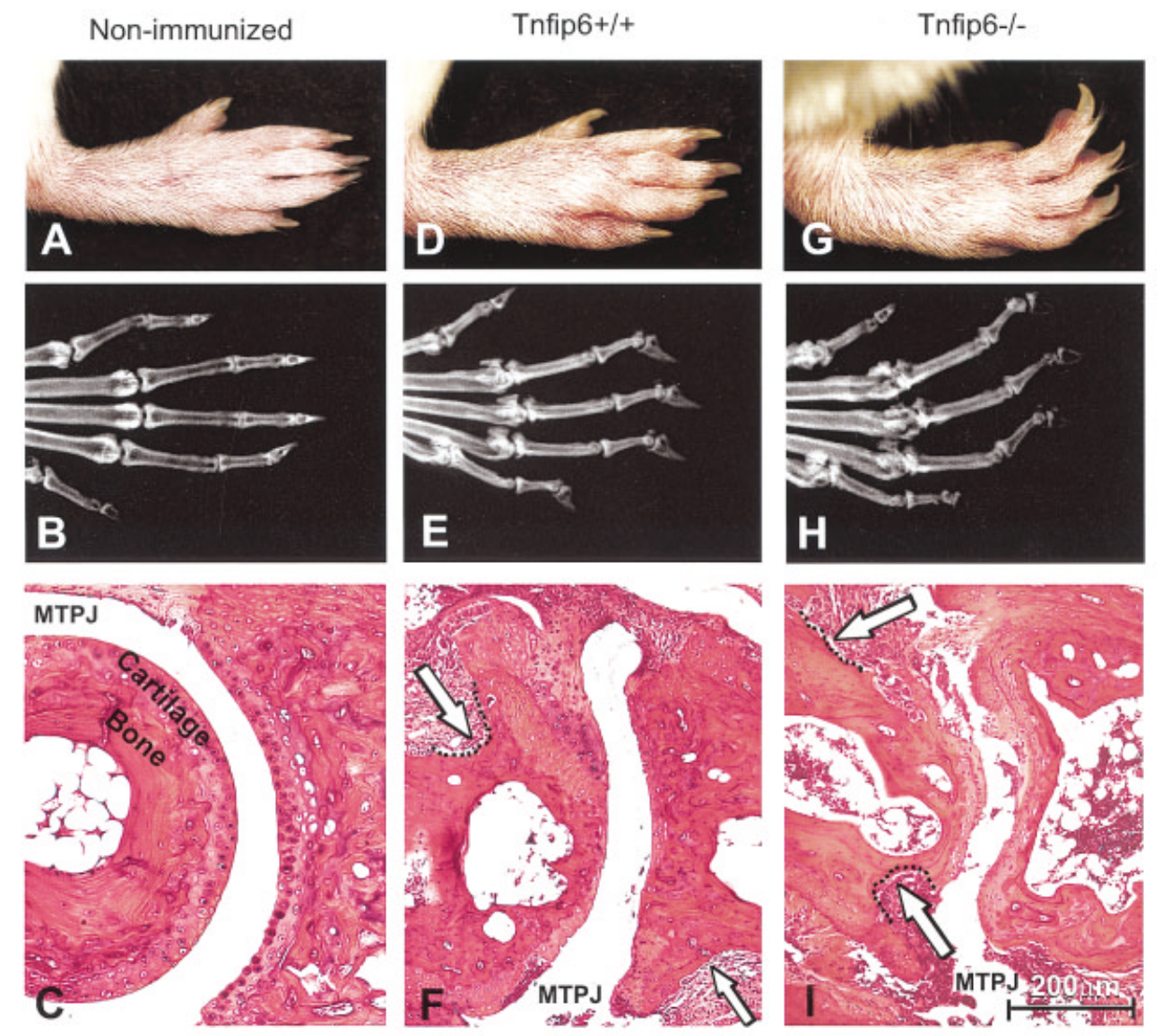

Figure 3. Macroscopic, radiographic, and histopathologic images of the hind paws of nonimmunized (A-C) and proteoglycan-immunized wild-type (D-F) and Tnfip6-deficient (G-I) BALB/c mice with arthritis. Macroscopic images, radiographs, and histologic sections were prepared on day 105 of the experiment ( $\sim 8$ weeks after the onset of the inflammation). The same paws are shown in the corresponding macroscopic pictures, radiographic images, and histologic sections. Joint deformities and cartilage and bone erosions are more pronounced in the Tnfip6-deficient mouse (G-I) than in the wild-type mouse (D-F), although the first clinical symptoms (redness and swelling of the paw) appeared on the same day in both mice. Hematoxylin and eosin-stained histologic sections show the second metatarsophalangeal joints (MTPJ) of the corresponding paws (C, F, and I). White arrows and dotted lines in $\mathbf{F}$ and $\mathbf{I}$ indicate invasion of the bone by pannus-like soft tissue. See Figure 1 for other definitions.

between the wild-type and Tnfip6-deficient animals in PG-specific T or B cell responses at any of those time points (test results on day 81 are shown in Figure 4). Stimulation of $\mathrm{T}$ cells (Figure 4A) and production of antigen-specific Th1- and Th2-type cytokines (Figure 4B), heteroantibodies (against human PG) (Figure 4C), and autoantibodies (against mouse PG) (Figure 4D) were all comparable in the 2 genotypes of mice.

Serum levels of the proinflammatory cytokines IL-1 $\beta$ (Figure 5A) and TNF $\alpha$ (Figure 5B) were also similar between the Tnfip6-null and wild-type animals.
However, serum concentrations of IL-6 (Figure 5C) and the acute-phase reactant SAA (Figure 5D) were significantly higher in the Tnfip6-deficient mice than in the wild-type mice at the acute phase of PGIA.

Enzyme activities in the joint tissues of Tnfip6deficient and wild-type BALB/c mice. Tnfip 6 binds $\mathrm{I} \alpha \mathrm{I}$, a powerful serine protease inhibitor in serum, and this interaction has been shown to potentiate the inhibitory effect of $\mathrm{I} \alpha \mathrm{I}$ on plasmin (9). Thus, plasmin activity was expected to be elevated in the joints with inflammation. Some plasmin activity was detected in the total extracts 
of nonarthritic joints (Figure 6A), which was most likely the result of an association with the normal metabolic turnover of matrix components. After the development of arthritis, however, plasmin activity was almost 2 -fold higher in the joint tissues of mice lacking Tnfip6, whereas only a moderate increase $(<50 \%)$ was found in the paws of wild-type animals (Figure 6A).

As an indicator of neutrophil invasion, the activity of the neutrophil granulocyte-specific enzyme myeloperoxidase was measured in the total extracts of nonarthritic and arthritic paws of wild-type and Tnfip6deficient animals (Figure 6B). Consistent with the histopathologic findings, myeloperoxidase activity was significantly elevated in the inflamed paws of Tnfip6-null mice when compared with the arthritic paws of wild-type animals (Figure 6B).
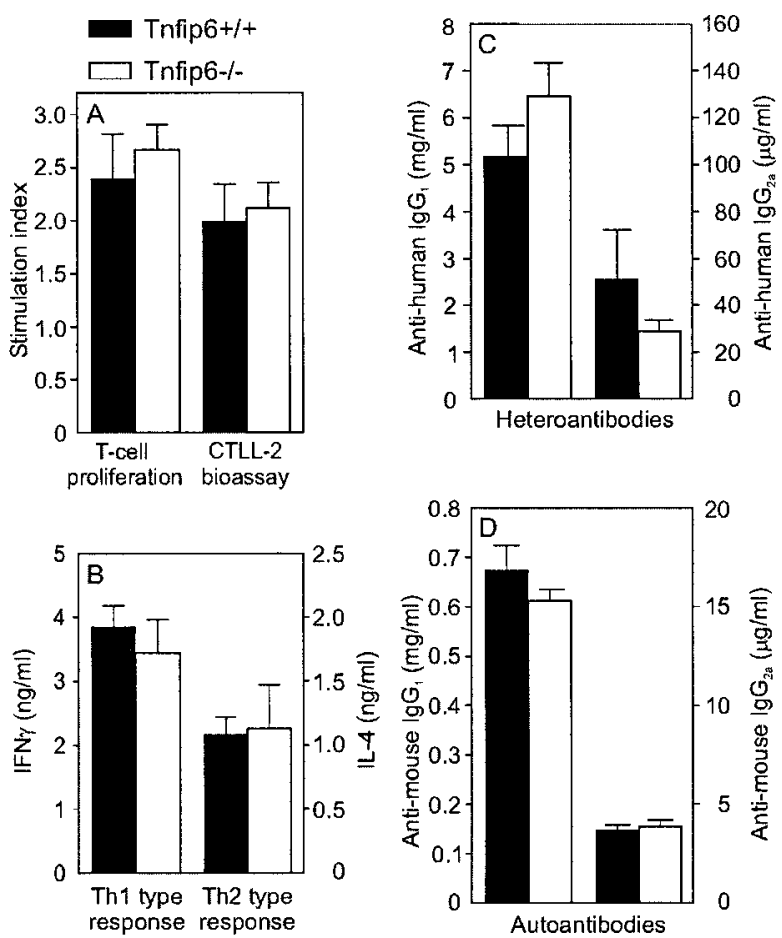

Figure 4. Summary of immune responses in wild-type $\left(\right.$ Tnfip $\left.6^{+/+}\right)$ and tumor necrosis factor $\alpha$-induced protein 6 (Tnfip6)-deficient $\left(\right.$ Tnfip $^{-1-}$ ) mice. Antigen (proteoglycan $[\mathrm{PG}]$ )-specific T cell proliferation and interleukin-2 (IL-2) production (A), and Th1-type cytokine interferon- $\gamma$ (IFN $\gamma)$ and Th2-type IL-4 (B) were measured in supernatants of PG-stimulated spleen cell cultures from arthritic mice. PG-specific antibodies against the immunizing human PG (C) and autoantibodies against mouse cartilage PG (D) (both the Th2supported IgG1 and Th1-supported IgG2a isotypes) were measured in the sera of animals at the end of the experiments. Bars show the mean and SEM in 35 Tnfip $6^{-1-}$ mice and 32 wild-type mice (same animals as shown in Figure 1).
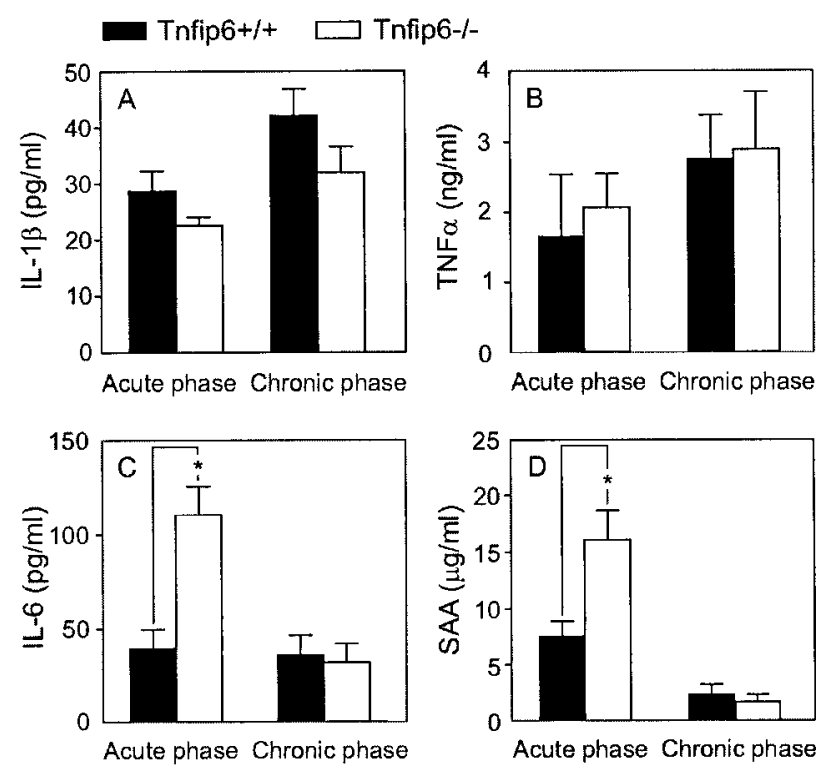

Figure 5. Serum concentrations of proinflammatory cytokines IL-1 $\beta$ (A), tumor necrosis factor $\alpha(\mathrm{TNF} \alpha)$ (B), and IL-6 (C) and the acute-phase protein serum amyloid A (SAA) (D). Cytokines were measured in serum samples of wild-type and Tnfip6-deficient BALB/c mice during the acute phase (on day 53) and chronic phase (on day 81) of proteoglycan-induced arthritis. Sera were collected from the retroorbital venous plexus of arthritic animals on day $53(\mathrm{n}=15$ in each group) or at the end of the experiments (day 81; $\mathrm{n}=35$ Tnfip $^{-/-}$mice and $\mathrm{n}=32$ wild-type mice, as in Figures 1 and 4). Bars show the mean and SEM. $*=P<0.01$ versus wild-type mice. See Figure 4 for other definitions.

We also determined the activity of the neutrophil elastase, which could be involved in tissue damage upon release from locally activated neutrophils. The activity of this enzyme was significantly higher in the arthritic paws of Tnfip6-knockout mice compared with that in the paws of the wild-type mice (Figure 6C), and this increase was proportional to the extent of myeloperoxidase activity (as measured by neutrophil number) in Tnfip6-null joints (Figure 6B).

Neutrophil influx into the peritoneal cavity during thioglycollate-induced peritonitis in wild-type and Tnfip6-deficient mice, and the effect of rMuTnfip6 treatment. The histopathologic examination of the joints (Figure 2) and the results of enzyme (myeloperoxidase and neutrophil elastase) assays (Figure 6) suggested that Tnfip6 deficiency could selectively facilitate the influx of neutrophil granulocytes into the site of inflammation. This was consistent with the observations of earlier studies in which it was reported that systemic or local administration of rHuTnfip6 inhibited neutrophil migration into nonspecific irritant- or cytokine-stimulated air 

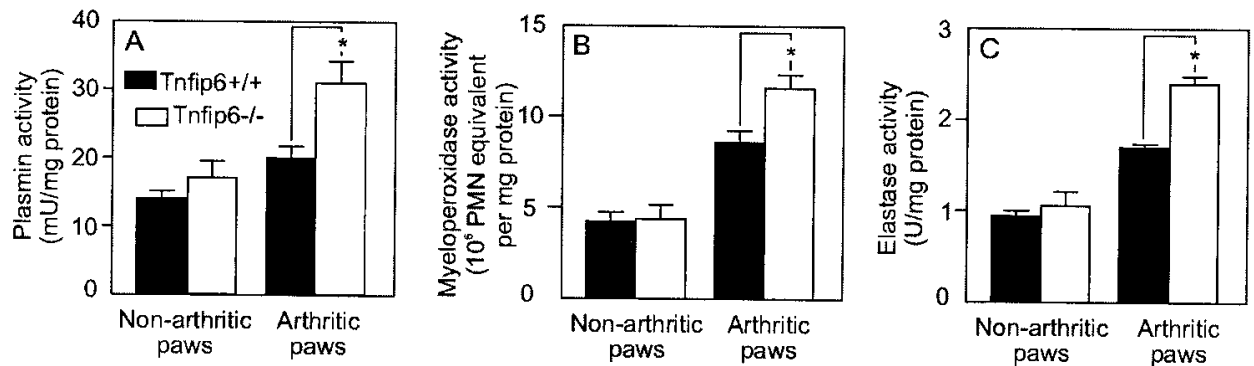

Figure 6. Activities of myeloperoxidase and 2 serine proteases measured in tissue extracts of the nonarthritic and inflamed paws of Tnfip6-deficient and wild-type BALB/c mice with proteoglycan-induced arthritis. Tissue extracts were prepared separately from the noninflamed and the inflamed paws of the same animal 2-4 days after the onset of arthritis. Arthritis scores of the inflamed paws ranged between 1 and 4 by this time point, and therefore equal numbers $(n=12)$ of arthritic paws with highly comparable individual arthritis scores were selected for these assays. The overall arthritis score was a mean \pm SEM $3.0 \pm 0.73$ in the wild-type mice and $2.91 \pm 0.71$ in the Tnfip6-deficient group. Enzyme activities were normalized to protein content. Myeloperoxidase activity (B) is expressed as the enzyme activity relative to the numbers of polymorphonuclear (PMN) cells (neutrophils) measured in peripheral blood of normal BALB/c mice, whereas the activity of plasmin (A) and neutrophil elastase $(\mathbf{C})$ is expressed in units, using the activity of the corresponding purified enzyme as a reference. $*=P<0.05$ versus wild-type mice. See Figure 4 for other definitions.

pouches in mice $(10,11)$. Since a method for the accurate measurement of leukocyte infiltration in the small peripheral joints of mice is not available, we used thioglycollate-induced sterile peritonitis to compare neutrophil accumulation in wild-type and Tnfip6deficient mice, and to quantitatively determine the effect of exogenous Tnfip6 on PMN cell extravasation.

This model is characterized by the influx of predominantly neutrophil granulocytes and monocytes/ macrophages into the peritoneal cavity $(26,27)$, from which these cells can be easily isolated. Indeed, the peritoneal exudate (lavage fluid) was dominated by PMN cells between 4 hours and 48 hours (showing a peak of influx at 24 hours) after injection of thioglycollate, whereas the number of mononuclear cells was relatively low and remained nearly constant during the 96-hour observation period in both genotypes of mice (Figure 7). At each time point between 4 hours and 96 hours, $\sim 2-3$ times more neutrophils migrated into the peritoneal cavities of Tnfip6-deficient animals than into the cavities of wild-type mice (Figure 7). A single dose $(30 \mu \mathrm{g})$ of rMuTnfip6, injected intravenously 20 minutes before the thioglycollate challenge, reduced the neutrophil numbers in the peritoneal lavage fluid by at least $50 \%$ in both wild-type and Tnfip6-deficient mice (Figure 7 , front columns), and this inhibitory effect on neutrophil influx was still detectable at 48 hours after thioglycollate administration to rMuTnfip6-injected animals.

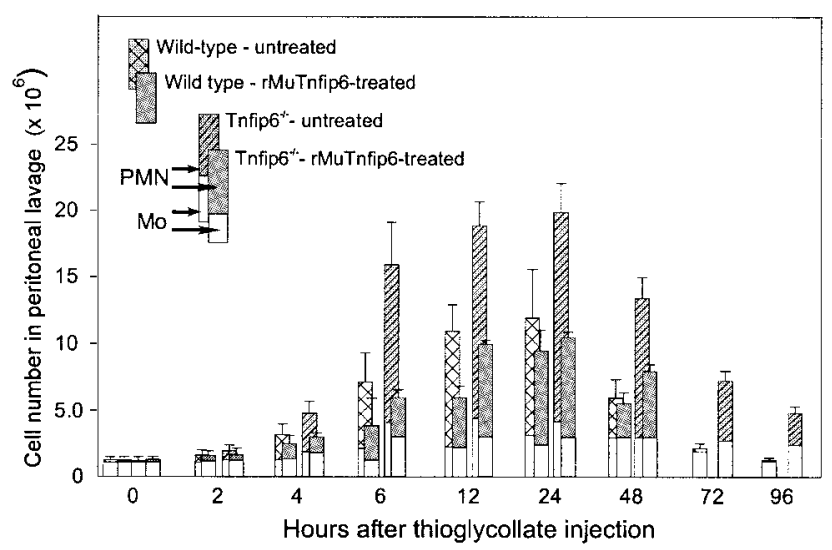

Figure 7. Neutrophil extravasation in thioglycollate-induced peritonitis in wild-type and Tnfip6-deficient mice, with or without treatment with recombinant murine Tnfip6 (rMuTnfip6). Columns at the back represent total cell numbers in the peritoneal cavity after $0.5 \mathrm{ml}$ of $4 \%$ thioglycollate injection, in which the upper areas correspond to neutrophil (polymorphonuclear [PMN]) cell number and the bottom areas are representative of the macrophage (monocyte [Mo]) numbers in the same samples. Columns at the front show the effect of rMuTnfip6 (30 $\mu \mathrm{g}$ injected intravenously 20 minutes before the thioglycollate challenge) on the peritoneal cell number. This intravenously injected rMuTnfip6, however, showed a rapid decline in the serum and was no longer detectable by enzyme-linked immunosorbent assay at 25-30 minutes after the injection (results not shown). Cells were counted in aliquots of the peritoneal lavage fluid, and differential counts were determined in May-Grünwald- and Giemsa-stained smears of the peritoneal lavage fluid and confirmed by flow cytometry. The proportion of lymphocytes (CD3+ and CD45/B220+ cells) was fewer than $2 \%$ of the total cell number at each time point (results not shown). Bars show the mean and SEM. See Figure 4 for other definitions. 


\section{DISCUSSION}

In this study, we demonstrate that Tnfip6 deficiency increases the severity and accelerates the progression of PGIA in BALB/c mice. Wild-type and Tnfip6deficient animals mount similar autoimmune responses (including antigen-specific $\mathrm{T}$ cell reactions, a balance between the Th1 and Th2 response, and antibody production) following immunization with cartilage PG. However, at the early (initial) phase of arthritis, there is a striking increase in the number of PMN cells in the synovium and joint exudate in mice lacking Tnfip6. Inflammatory destruction of the articular cartilage, erosion of bone, and joint deformities develop earlier and are more extensive in Tnfip6-null mice than in wild-type $\mathrm{BALB} / \mathrm{c}$ mice. Serum markers of inflammation, including IL-6 and SAA, are also significantly elevated in Tnfip6-deficient mice during the acute phase of PGIA. Thus, although treatment of mice with recombinant Tnfip6 has been shown to exert a therapeutic effect in various forms of experimentally induced inflammation $(6,7,10,11)$, the lack of endogenous Tnfip6 increases the severity of inflammation.

In vivo expression of TSG-6/Tnfip6 has been associated with inflammatory arthritis $(3,5)$, but the protein is produced in a variety of cells exposed to proinflammatory stimuli in vitro $(2,28,29)$, and also in a physiologic process such as matrix formation around the cumulus cell-oocyte complex (30). In fact, the relationship between impaired cumulus cell-oocyte matrix assembly and female sterility in Tnfip6-knockout mice reveals an essential role for this protein in the formation of crosslinked HA fibers around the oocyte that is necessary for the expansion of cumulus matrix and subsequent oocyte fertilization (15).

The antiinflammatory properties of Tnfip6 have been mainly attributed to its ability to potentiate the antiplasmin activity of $\mathrm{I} \alpha \mathrm{I}$ upon association with this protease inhibitor, leading to the subsequent downregulation of the activity of a number of matrixdegrading proteases (7-10). However, a recent study (11) found that the isolated Link module domain of Tnfip6, which lacks the ability to associate with $\mathrm{I} \alpha \mathrm{I}$, could still inhibit the influx of neutrophils to the site of inflammation. This observation suggests that Tnfip6 might exert antiinflammatory effects in an I $\alpha \mathrm{I}$ independent manner.

Although we have found elevated plasmin activity in tissue extracts prepared from the arthritic joints of Tnfip6-null mice, the most striking effects of Tnfip6 deficiency are the increases in the number of neutrophils and the activity of neutrophil-derived enzymes in the affected joints. Accelerated destruction of the articular cartilage and rapid progression of joint deformities in Tnfip6-null mice, therefore, could be associated with both enhanced neutrophil invasion and insufficient protection of cartilage matrix components from plasminactivated proteases. We have shown that influx of PMN cells into the peritoneal cavity is also enhanced in Tnfip6-null mice in response to local injection of thioglycollate, indicating that neutrophil egress is facilitated in the absence of Tnfip6, independently of the site or type of inflammation.

Tnfip6 is an HA binding protein, and HA is known to support the CD44-dependent rolling of leukocytes (31-34), the first step toward their extravasation (35). It is a natural question, therefore, whether Tnfip6 is somehow involved in the adhesive interaction between CD44 and HA. A recent study by our group (36) demonstrated that, indeed, Tnfip6 modulates the interaction between HA and cell-surface CD44. Binding of HA to the cell surface is enhanced when CD44-positive cells are exposed to preformed Tnfip6-HA complexes, and HA in these complexes is recognized by cells expressing inactive CD44 that does not bind HA constitutively, i.e., without induction (36). Cells roll more readily on immobilized substrates consisting of Tnfip6-HA complexes as compared with substrates of HA alone, but firm adhesion to HA is not significantly facilitated in the presence of Tnfip6. Importantly, cellsurface-bound Tnfip6-HA complexes prevent CD44mediated leukocyte adhesion to HA (36). Although these in vitro observations do not provide a full explanation for the in vivo effects of Tnfip6 (or the lack thereof), intravital videomicroscopy has currently revealed some important differences between Tnfip6deficient and rMuTnfip6-treated mice. As shown for thioglycollate-induced peritonitis in this study, intravenously injected rMuTnfip6 almost completely abolishes leukocyte extravasation in $\mathrm{TNF} \alpha$-induced local ear inflammation (see ref. 37 and Szántó S, et al: unpublished observations).

The results of these in vivo experiments suggest that Tnfip6 plays a role in the control of leukocyte influx into arthritic joints (most likely via interference with CD44/HA-mediated adhesion events), and is involved locally in the protection of cartilage matrix from proteolytic damage through complex formation with the serine protease inhibitor $\mathrm{I} \alpha \mathrm{I}(7,8)$. The most important message of our in vitro and in vivo studies is that Tnfip6 can directly modulate cell adhesion $(36,37)$, PMN cell extravasation (Figures 2 and 7), and perhaps also, the 
production of proinflammatory mediators such as IL-6 (Figure 5C). Thus, the suppressive effects of Tnfip6 on arthritis $(6,7)$ are exerted through more than one mechanism. The effects of Tnfip6 are not restricted to joints (and arthritis), since this protein seems to inhibit other types of inflammation as well. Inhibition of inflammatory leukocyte (neutrophil) influx into the joint could be the most important function of Tnfip6 in arthritis suppression, but its interaction with $\mathrm{I} \alpha \mathrm{I}$ might provide the cartilage matrix an enhanced protection from proteolytic damage. Therefore, in inflammatory/rheumatoid arthritis, recombinant Tnfip6 holds promise as a therapeutic agent.

\section{ACKNOWLEDGMENTS}

The authors thank Vyacheslav A. Adarichev, Csaba Vermes, Andrew B. Nesterovitch, Andrea Gonda, Bara Sarraj, and Rajesh V. Kamath for fruitful discussions and help with the experiments, and Stiliani Christodoulu, Kevin Kolman, and Sonja Velins for expert technical assistance.

\section{REFERENCES}

1. Lee TH, Lee GW, Ziff EB, Vilcek J. Isolation and characterization of eight tumor necrosis factor-induced gene sequences from human fibroblasts. Mol Cell Biol 1990;10:1982-8.

2. Lee TH, Wisniewski HG, Vilcek J. A novel secretory tumor necrosis factor-inducible protein (TSG-6) is a member of the family of hyaluronate binding proteins, closely related to the adhesion receptor CD44. J Cell Biol 1992;116:545-57.

3. Wisniewski HG, Maier R, Lotz M, Lee S, Klampfer L, Lee TH, et al. TSG-6: a TNF-, IL-1-, and LPS-inducible secreted glycoprotein associated with arthritis. J Immunol 1993;151:6593-601.

4. Fulop C, Kamath RV, Li Y, Otto JM, Salustri A, Olsen BR, et al. Coding sequence, exon-intron structure and chromosomal localization of murine TNF-stimulated gene 6 that is specifically expressed by expanding cumulus cell-oocyte complexes. Gene 1997;202:95-102.

5. Bayliss MT, Howat SL, Dudhia J, Murphy JM, Barry FP, Edwards $\mathrm{JC}$, et al. Up-regulation and differential expression of the hyaluronan-binding protein TSG-6 in cartilage and synovium in rheumatoid arthritis and osteoarthritis. Osteoarthritis Cartilage 2001; 9:42-8.

6. Mindrescu C, Thorbecke GJ, Klein MJ, Vilcek J, Wisniewski HG. Amelioration of collagen-induced arthritis in DBA/1J mice by recombinant TSG-6, a tumor necrosis factor/interleukin1-inducible protein. Arthritis Rheum 2000;43:2668-77.

7. Bardos T, Kamath RV, Mikecz K, Glant TT. Anti-inflammatory and chondroprotective effect of TSG-6 (tumor necrosis factor- $\alpha$ stimulated gene-6) in murine models of experimental arthritis. Am J Pathol 2001;159:1711-21.

8. Glant TT, Kamath RV, Bardos T, Gal I, Szanto S, Murad YM, et al. Cartilage-specific constitutive expression of TSG-6 protein (product of tumor necrosis factor $\alpha$-stimulated gene 6) provides a chondroprotective, but not antiinflammatory, effect in antigeninduced arthritis. Arthritis Rheum 2002;46:2207-18.

9. Wisniewski HG, Burgess WH, Oppenhein JD, Vilcek J. TSG-6, an arthritis-associated hyaluronan binding protein, forms a stable complex with the serum protein inter- $\alpha$-inhibitor. Biochemistry 1994:33:7423-9.
10. Wisniewski HG, Hua JC, Poppers DM, Naime D, Vilcek J, Cronstein BN. TNF/IL-1-inducible protein TSG-6 potentiates plasmin inhibition by inter- $\alpha$-inhibitor and exerts a strong antiinflammatory effect in vivo. J Immunol 1996;156:1609-15.

11. Getting SJ, Mahoney DJ, Cao T, Rugg MS, Fries E, Milner CM, et al. The link module from human TSG-6 inhibits neutrophil migration in a hyaluronan- and inter- $\alpha$-inhibitor-independent manner. J Biol Chem 2002;277:51068-76.

12. Glant TT, Mikecz K, Arzoumanian A, Poole AR. Proteoglycaninduced arthritis in BALB/c mice: clinical features and histopathology. Arthritis Rheum 1987;30:201-12.

13. Mikecz K, Glant TT, Poole AR. Immunity to cartilage proteoglycans in BALB/c mice with progressive polyarthritis and ankylosing spondylitis induced by injection of human cartilage proteoglycan. Arthritis Rheum 1987;30:306-18.

14. Glant TT, Finnegan A, Mikecz K. Proteoglycan-induced arthritis: immune regulation, cellular mechanisms and genetics. Crit Rev Immunol 2003;23:199-250.

15. Fulop C, Szanto S, Mukhopadhyay D, Bardos T, Kamath RV, Rugg MS, et al. Impaired cumulus mucification and female sterility in tumor necrosis factor-induced protein-6 deficient mice. Development 2003;130:2253-61.

16. Otto JM, Chandrasekaran R, Vermes C, Mikecz K, Finnegan A, Rickert SE, et al. A genome scan using a novel genetic cross identifies new susceptibility loci and traits in a mouse model of rheumatoid arthritis. J Immunol 2000;165:5278-86.

17. Adarichev VA, Valdez JC, Bardos T, Finnegan A, Mikecz K, Glant TT. Combined autoimmune models of arthritis reveal shared and independent qualitative (binary) and quantitative trait loci. J Immunol 2003;170:2283-92.

18. Nentwich HA, Mustafa Z, Rugg MS, Marsden BD, Cordell MR, Mahoney DJ, et al. A novel allelic variant of the human TSG-6 gene encoding an amino acid difference in the CUB module: chromosomal localization, frequency analysis, modeling, and expression. J Biol Chem 2002;277:15354-62.

19. Lesley J, English NM, Gal I, Mikecz K, Day AJ, Hyman R. Hyaluronan binding properties of a CD44 chimera containing the link module of TSG-6. J Biol Chem 2002;277:26600-8.

20. Glant TT, Cs-Szabo G, Nagase H, Jacobs JJ, Mikecz K. Progressive polyarthritis induced in $\mathrm{BALB} / \mathrm{c}$ mice by aggrecan from human osteoarthritic cartilage. Arthritis Rheum 1998;41:1007-18.

21. Hanyecz A, Berlo SE, Szanto S, Broeren CP, Mikecz K, Glant TT. Achievement of a synergistic adjuvant effect on arthritis induction by activation of innate immunity and forcing the immune response toward the Th1 phenotype. Arthritis Rheum 2004;50:1665-76.

22. Mikecz K, Brennan FR, Kim JH, Glant TT. Anti-CD44 treatment abrogates tissue edema and leukocyte infiltration in murine arthritis. Nat Med 1995;1:558-63.

23. Bardos T, Mikecz K, Finnegan A, Zhang J, Glant TT. T and B cell recovery in arthritis adoptively transferred to SCID mice: antigenspecific activation is required for restoration of autopathogenic CD4+ Th1 cells in a syngeneic system. J Immunol 2002;168: 6013-21.

24. Cao T, Pinter E, Al Rashed S, Gerard N, Hoult JR, Brain SD. Neurokinin-1 receptor agonists are involved in mediating neutrophil accumulation in the inflamed, but not normal, cutaneous microvasculature: an in vivo study using neurokinin-1 receptor knockout mice. J Immunol 2000;164:5424-9.

25. De Santi MM, Martorana PA, Cavarra E, Lungarella G. Pallid mice with genetic emphysema: neutrophil elastase burden and elastin loss occur without alteration in the bronchoalveolar lavage cell population. Lab Invest 1995;73:40-7.

26. Green AP, Mangan F, Ormerod JE. Induction of cell infiltration and acid hydrolase release into the peritoneal cavity of mice. Inflammation 1980;4:205-13.

27. Henderson RB, Hobbs JA, Mathies M, Hogg N. Rapid recruit- 
ment of inflammatory monocytes is independent of neutrophil migration. Blood 2003;102:328-35.

28. Bandman O, Coleman RT, Loring JF, Seilhamer JJ, Cocks BG. Complexity of inflammatory responses in endothelial cells and vascular smooth muscle cells determined by microarray analysis. Ann N Y Acad Sci 2002;975:77-90.

29. Fessler MB, Malcolm KC, Duncan MW, Worthen GS. A genomic and proteomic analysis of activation of the human neutrophil by lipopolysaccharide and its mediation by p38 mitogen-activated protein kinase. J Biol Chem 2002;277:31291-302.

30. Mukhopadhyay D, Hascall VC, Day AJ, Salustri A, Fulop C. Two distinct populations of tumor necrosis factor-stimulated gene-6 protein in the extracellular matrix of expanded mouse cumulus cell-oocyte complexes. Arch Biochem Biophys 2001;394:173-81.

31. DeGrendele HC, Estess P, Picker LJ, Siegelman MH. CD44 and its ligand hyaluronate mediate rolling under physiologic flow: a novel lymphocyte-endothelial cell primary adhesion pathway. J Exp Med 1996;183:1119-30.

32. Gal I, Lesley J, Ko W, Gonda A, Stoop R, Hyman R, et al. Role of the extracellular and cytoplasmic domains of CD44 in the rolling interaction of lymphoid cells with hyaluronan under physiologic flow. J Biol Chem 2003;278:11150-8.

33. DeGrendele HC, Estess P, Siegelman MH. Requirement for CD44 in activated $\mathrm{T}$ cell extravasation into an inflammatory site. Science 1997;278:672-5.

34. Siegelman MH, Stanescu D, Estess P. The CD44-initiated pathway of T-cell extravasation uses VLA-4 but not LFA-1 for firm adhesion. J Clin Invest 2000;105:683-91.

35. Springer TA. Traffic signals for lymphocyte recirculation and leukocyte emigration: the multistep paradigm. Cell 1994;76: 301-14.

36. Lesley J, Gal I, Mahoney DJ, Cordell MR, Rugg MS, Hyman R, et al. TSG-6 modulates the interaction between hyaluronan and cell surface CD44. J Biol Chem 2004;279:25745-54.

37. Szanto S, Bardos T, Kolman KJ, Gonda A, Gal I, Glant TT, et al. TSG-6 (TNF $\alpha$-stimulated gene-6)-deficiency accelerates inflammatory events, cartilage damage and bone erosion in a murine model of progressive polyarthritis [abstract]. Arthritis Rheum 2003;48 Suppl 9:S252. 